\title{
Defining functional brain networks using unsupervised density-peak clustering
}

\author{
Ori Ossmy ${ }^{1}$, Roy Mukamel ${ }^{2}$
}

${ }^{1}$ Department of Psychology and Center of Neural Science, New York University

2School of Psychology and Sagol School of Neuroscience, Tel Aviv University

\section{Contact Information}

Roy Mukamel

School of Psychological Sciences \& Sagol School of Neuroscience

Tel-Aviv University, Tel-Aviv, 69978, Israel

+972-3-6407246

Email: rmukamel@tau.ac.il 


\begin{abstract}
Background: Parcellating the human brain into areas based on their neural connectivity is essential for understanding the functional organization of neural networks.

New Method: We adapted density-peak clustering to identify functional neural networks in individuals without the need to select seed regions. We first assess the similarity between each pair of voxels based on their activation time-courses and then aggregate the voxels based on the assumption that cluster centers are dense (have high similarity with many voxels) and are at large distance from other high-density voxels. This data-driven approach allows intuitive selection of cluster centroids in individual subjects.

Results: We applied our approach on resting-state data of individual subjects. Although similar networks across subjects were identified, there was large variability in the number of networks and their anatomical distribution between subjects. Manipulating the main free parameter of the model (density level threshold) revealed a hierarchic representation in which large clusters are divided to smaller sub-clusters when decreasing the threshold.

Comparison with Existing Method: To date, most connectiviy-based parcellations begin with selecting an initial seed region and are therefore limited and heavily reliant on prior theoretical knowledge. Existing methods also require many pre-defined parameters and were usually used at the group level.

Conclusions: Adapting density-peak clustering algorithm to neural data has potential implications for understanding individual differences in functional networks without predetermining the number of networks or functional/anatomical definition of a seed region. This data-driven approach may pave the way to deeper investigation of the brain structure-function relationship within individual humans.
\end{abstract}

Keywords: clustering, functional connectivity, fMRI; resting-state networks. 


\section{Introduction}

The human brain consists of multiple cortical and sub-cortical areas that manifest structural and functional interactions (Zilles and Amunts, 2010). In the last two decades, neuroscientists are interested in understanding how the brain is parcellated to functional networks and how these networks relate to brain structure. To this end, studies have measured the functional similarity between anatomically separate brain regions by examining the level of co-activation of spontaneous time-series recorded during rest (Van Den Heuvel and Pol, 2010).

Many approaches to functional brain parcellation have been proposed and most of them rely on pre-selection of "seed regions" (Wig et al., 2014). In these approaches, the parcellation process traditionally begins with extraction of the neural signal from a certain anatomical region ("seed region"), and the correlation of this signal with other voxels across the brain is calculated. Finally, voxels that are most correlated to the seed region are identified as part of a common functional network. To identify different functional networks, this processes needs to be repeated multiple times, each time with a different seed region.

Seed-region approaches are effective in identifying consistent functional networks that were replicated across various datasets (Power et al., 2011) and correspond with task response patterns (Smith et al., 2009). However, these approaches are limited because the initial choice of seed region needs to be based on prior knowledge about the brain areas (from previous studies or a brain atlas; Hagmann et al., 2008). Consider the case of using the amygdala as seed region. Presumably, the amygdala is not a homogeneous region and can be split to smaller subunits when parcellating the entire brain to functional networks. For example, one subunit might belong to one functional network (e.g. the centromedial amygdala is connected to default mode network) and another subunit may belong to a different functional network (e.g., the dorsal amygdala is connected to fronto-parietal networks). Yet, such different functional networks would be missed in lack of prior knowledge if the amygdala is defined as one seed region based on anatomical criteria alone.

Some studies take a data-driven approach by using unsupervised clustering techniques as k-means or independent component analysis (ICA; Damoiseaux et al., 2006; Esposito et al., 2005). In these approaches, the functional connections between all voxels in the brain are taken into account and clustering is used to find an underlying structure. Yet, these approaches are usually derived either from average data across many individuals or pre-defined algorithm parameters that are identical for all subjects (e.g., number of networks when using k-means clustering). Therefore, these approaches lack the ability to achieve data-driven individual-level precision or account for functional difference between subjects (Sohn et al., 2015).

Here, we adapted a recent density-peak clustering algorithm (Rodriguez and Laio, 2014) to neural data in a way that allows parcellation of voxels to functional networks in a data-driven manner without prior need of defining a particular seed region or the number of networks in the data (see Fig. 1). The proposed approach is based on the assumption that cluster centers are (1) dense and surrounded by neighbors (that is, voxels with similar neural activation) with lower density; and (2) have a relatively large distance from other cluster centers with a higher density. Previous studies have shown this method to be highly efficient for varied datasets including several graphic distributions, face recognition, and spike sorting (Qiu et al., 2017; Rodriguez and Laio, 2014).

The current study is a unique implementation of this method on resting-state functional magnetic resonance imaging (fMRI) data from the human brain. It holds an interesting potential as it is entirely data-driven and can be used as a tool to examine individual differences in functional networks. We show the potential of our approach in the context of resting state data and illustrate that the main pre-defined free parameter is informative with respect to the problem 
of selecting number of clusters for each individual subject and characterizing functional networks.

\section{Materials and Methods}

All analysis codes are shared in GitHub: https://anonymous.4open.science/r/f1bac9fdda6d-458c-965d-f127224725cc/

\section{Resting-state data}

We used an open source dataset downloaded from the NITRC project (Neuroimaging Informatics Tools and Resources; https://www.nitrc.org/frs/?group_id=296). The dataset contains 23 subjects (15 female; ages=20-40). The Blood oxygenation level dependent (BOLD) data during resting state was obtained on a 3T General Electric scanner with an 8 channel head coil recorded by Pekar J.J. and Mosofsky S.H. An echo-planar imaging sequence was used to obtain the functional data (TR $=2500 \mathrm{msec}$; number of timepoints=123). In addition, anatomical reference was obtained by $\mathrm{T} 1$-weighted scan $($ voxel size $=1 \times 1 \times 1 \mathrm{~mm})$ per subject.

\section{Data preprocessing}

All fMRI data were processed using the BrainVoyager QX software (version 2.6, Brain Innovation, Maastricht, Netherlands). A preprocessing procedure was performed on all functional images and included cubic spline slice-time correction, trilinear 3D motion correction, and high-pass filtering (above $.006 \mathrm{~Hz}$ ). Additionally, we assessed head movements and excluded scans with head movement exceeding $2 \mathrm{~mm}$. The 2D functional images were coregistered to the anatomical images. Functional data of individual subjects was spatially smoothed using Gaussian filter (FWHM 6mm).

\section{Density-peak Clustering}

Preprocessed data was analyzed voxel-wise at the individual subject level. For each subject, a distance matrix between all pairs of voxels was calculated using correlation distance:

$$
\mathrm{d}_{i, j}=1-\frac{\sum_{k=1}^{n}\left(x_{i_{k}}-\bar{x}_{i}\right) \cdot \sum_{k=1}^{n}\left(x_{j_{k}}-\bar{x}_{j}\right)}{\sqrt{\sum_{k=1}^{n}\left(x_{i_{k}}-\bar{x}_{i}\right)^{2}} \cdot \sqrt{\sum_{k=1}^{n}\left(x_{j_{k}}-\bar{x}_{j}\right)^{2}}}
$$

Where

$$
\begin{aligned}
& \bar{x}_{i}=\frac{1}{n} \cdot \sum_{k=1}^{n} x_{i_{k}} \\
& \bar{x}_{j}=\frac{1}{n} \cdot \sum_{k=1}^{n} x_{j k}
\end{aligned}
$$

i and $j$ signify two different voxels, $n=123$ is the number of points in the time-series (volumes), and $x$ is the $\mathrm{AMRI}$ signal value. For each voxel, we extracted the raw BOLD timecourse and calculated its distance with all other voxels (see Fig 1. for sample matrix on 15 voxels and a multi-dimensional scaling visualization of this matrix). Next, for each voxel we calculated two measures: density and distance. A density coefficient $\rho$ was estimated such that

$$
\rho_{i}=\sum_{j=1}^{m} \chi\left(d_{i j}-d_{c}\right)
$$

Where $X(x)=1$ if $x<0$ and $X(x)=0$ otherwise, and $m$ is the number of voxels. $d_{c}$ represents the cutoff distance and is the main free parameter of the method.,. Thus, $\rho$ is a density measure 
for each voxel, corresponding to the number of voxels that have a distance that is lower than a certain pre-defined cutoff distance. In principle, the cutoff distance can be determined from the dataset by comparing the entropy values for different distance cutoffs $d_{\mathrm{c}}$. The most reasonable $d_{c}$ is the one that provided the lowest value of entropy (for full details, see Wang et al., 2015). In the current study, we examined how the cutoff distance affects the number, and definition of brain networks.

Once voxel densities are calculated, we compute a cluster distance coefficient $\delta$ as a second measure according to the following formula:

$$
\delta_{i}=\min _{j: \rho_{j}>\rho_{i}}\left(d_{i j}\right)
$$

$\delta$ is the minimal distance between each voxel and the next voxel with a higher density. For the point with the highest density, we conventionally set: $\delta_{i}=\max \left(d_{i j}\right)$ (the maximum distance value). The distance and density values were then multiplied to create a $y$ score for each voxel. High $y$ scores correspond to voxels with high density and also a large distance from other voxels with higher density, and therefore constitute potential cluster centroids (functional network hubs). We arbitrarily defined outliers as voxels with $\gamma>9$ SD above the mean $\gamma$. Outliers represent voxels for which the timecourse is a cluster centroid (points of highest density and distance from other centroids). The number of outliers determined the number of clusters (functional networks) identified. After cluster centroids were identified, each voxel in the brain is assigned to the closest cluster centroid.

\section{Clusters Robust Core}

The algorithm does not have noise-signal cutoff, therefore each voxel in the brain is assigned to a certain cluster. However, it is possible to define a border for each cluster, and avoid inclusion of noisy voxels that have low correlation values with all cluster centroids. This border is defined as correlation values outside the $d_{c}$ of the clusters centroids. We denote this border as the cluster's robust core.

\section{Results}

\section{Functional networks in individual Subjects}

Individual brains were masked to use only voxels containing gray matter ( $\mathrm{M}=45791$ voxels across subjects)We first demonstrate that functional networks, based on resting-state data can be strikingly different between subjects. Fig. 2a demonstrates clustering results of two different subjects (each cluster is a different color) using a cutoff distance $d_{c}$ equal to $15 \%$ of the mean correlation distance across all voxel pairs in the brain. The mean number of networks identified across subjects $(N=23)$ ranged from 4 to 16 . The size of the clusters ranged from 439 to 11310 voxels (across clusters and subjects). Despite high inter-individual variability, we see common and established networks in all subjects corresponding to the default network, posterior networks containing the occipital cortex, midline network containing the precuneus and an anterior pole of the prefrontal lobe as well as parietal regions. We also find high symmetry between the two hemispheres in all subjects.

\section{Cutoff Distance}

There is only one free parameter in implementing the proposed algorithm and this is the cutoff distance $d_{c}$. We tested the impact of this parameter on clustering, both quantitatively (Fig. 2b) and qualitatively (Fig. 2c) by systematically varying its value (using cutoff distance ranging from $0.5 \%$ to $150 \%$ of the mean whole brain correlation (Fig. $2 \mathrm{~b}$ left panel). The maximal number of clusters in all subjects is obtained when cutoff $=5 \%$. We see that the number of 
clusters decreases as we raise the cutoff above $5 \%$. This is not surprising since increasing the cutoff means using a higher radius for the density calculation which results in overall higher density values $(\rho)$ and reduced variability across voxels. This reduction in density variability leads to similar $\delta$ values and lower number of detected centroids (outliers). Similarly, reducing the cutoff (in our case below $5 \%$ of the mean correlation) also resulted in lower number detected clusters. Low cutoff reduces the overall density of all voxels and variability across voxels. Once again, such reduction in variability would lead to similar $\gamma$ values, and lower number of detected clusters centroids.

In terms of $\delta$ - we found that the choice of cutoff parameter had little influence. However, we did find high variability across subjects in this feature, implying that this feature is the basis for inter-individual differences in number of clusters.

\section{Hierarchical Clustering}

Qualitatively, we found that increasing cutoff distance aggregates clusters obtained when clustering with smaller cutoffs (Fig. 2c). This creates a hierarchical parcellation in all the subjects even though there is high inter-subject variability. This can be attributed to the more liberal definition of "neighbors" used in the density calculations, so that increasing cutoff results in higher $\rho$ values and aggregates other voxels that were otherwise centroids or part of a different network when smaller cutoffs were used.

\section{Robust Core Maps}

Finally, we explored the robust core maps for each subject at different cutoffs. Fig. $2 d$ demonstrates these maps in the same subjects and cutoffs as in Fig. 2c. Thus, the colored maps in Fig. 2d represent different functional networks, and the size of each network is limited to voxels that are inside the selected radius $\left(d_{c}\right)$ of the cluster center. In this approach, not all voxels are necessarily assigned to a cluster - thus reducing potential effects of noise (i.e. voxels that are functionally far away from identified networks).

\section{Discussion}

In this study, we used a novel data-driven approach by applying density-peaks clustering on time series correlation between voxels. To date, fMRI clustering methods are limited in accuracy and reliability at the single-subject level (Van Den Heuvel and Pol, 2010). Unlike traditional methods, our approach is not biased by prior knowledge of anatomy, regions of interest or number of pre-conceived hypothetical networks. It provides a simple and intuitive technique to define functional networks at the individual subject level using density maxima.

Our findings show that the proposed algorithm is effective in capturing neural networks based on the individual subject's data during resting-state. Future studies can further apply density-peak clustering on data recorded during different tasks for characterizing functional systems at the individual level.

Our data show that despite network commonalities, the number and extent of functional networks is highly variable across individual subjects. Therefore, identifying neural networks based on group data neglects the immense individual differences in both brain anatomy and functional connectivity. This may limit understanding the coherence between anatomical and functional parcellation of the human brain since high coherence at the group level does not necessarily reflects high coherence in individuals and vice versa. We suggest density-peak clustering can serve as a technique for a hierarchical functional parcellation of the brain at the individual level which is a critical step towards deeper understanding of the structure-function relationship in the brain.

Finally, there are several ways in which our single subject approach could be extended to the group level if needed (e.g. time course concatenation or time course averaging). Thus, 
this approach can be used for creating parcellation markers for sub-types of psychological profiles and can advance our understanding of individual differences in diverse pathologies.

In sum, our findings add to a growing body of recent research on the functional organization of neural networks in the brain (Herbet and Duffau, 2020; Ipiña et al., 2019; Ji et al., 2019) by providing a framework for neuroscientists who are interested in explaining individual differences by examining the organization of brain networks.

\section{Acknowledgements}

This study was supported by the I-CORE Program of the Planning and Budgeting Committee and the Israel Science Foundation (grants No. 1771/13 and 2043/13) and Human Frontiers Science Project (HFSP) Career Development Award (CDA00078/2011-C) to RM; The Yosef Sagol Scholarship for Neuroscience Research, The Israeli Presidential Honorary Scholarship for Neuroscience Research and the Sagol School of Neuroscience fellowship to OO. The funders had no role in study design, data collection and analysis, decision to publish, or preparation of the manuscript. 


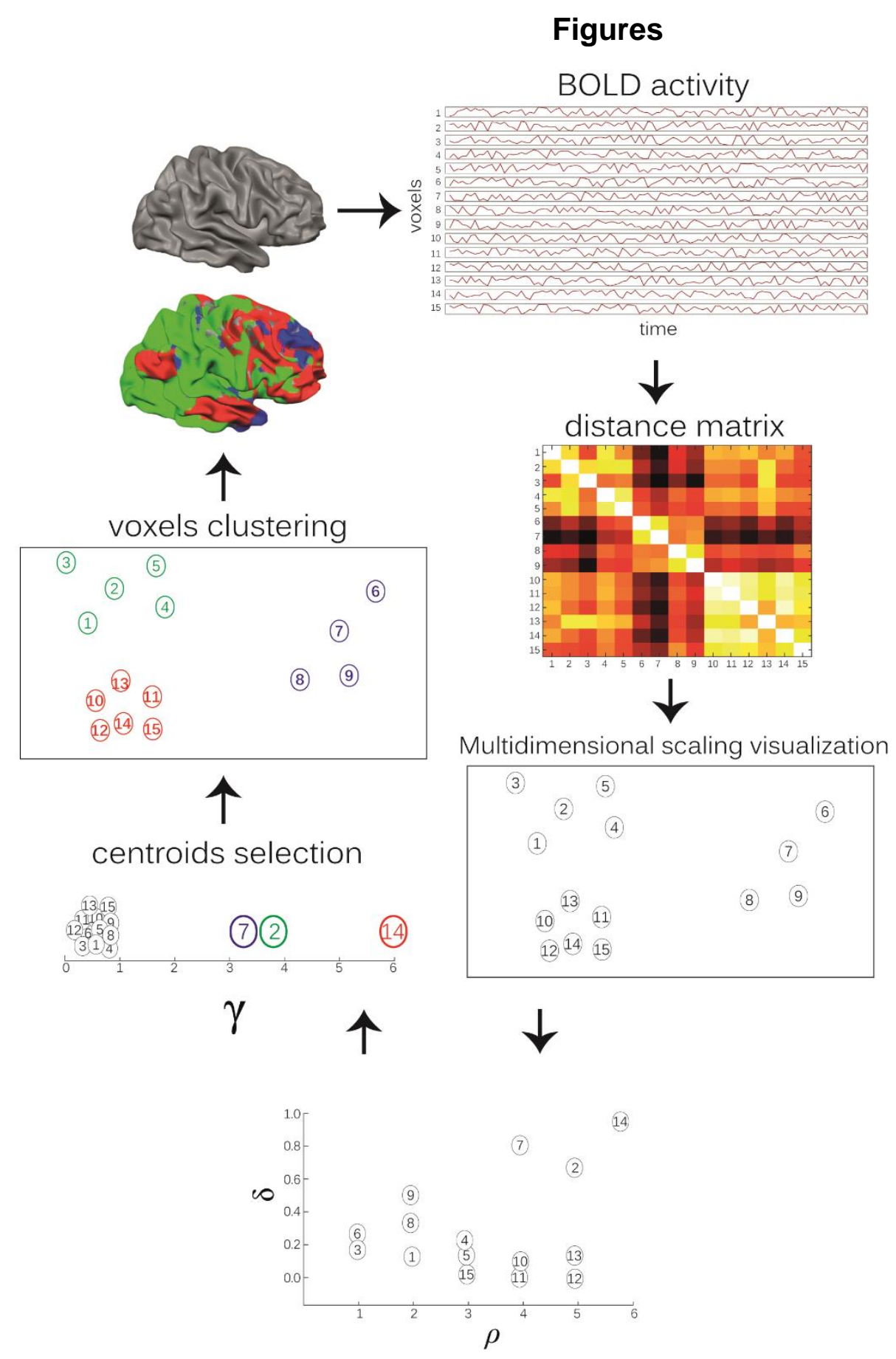

Fig. 1. General description of the approach for the characterization and clustering of voxels at the single subject level (See Materials and Methods). 
a
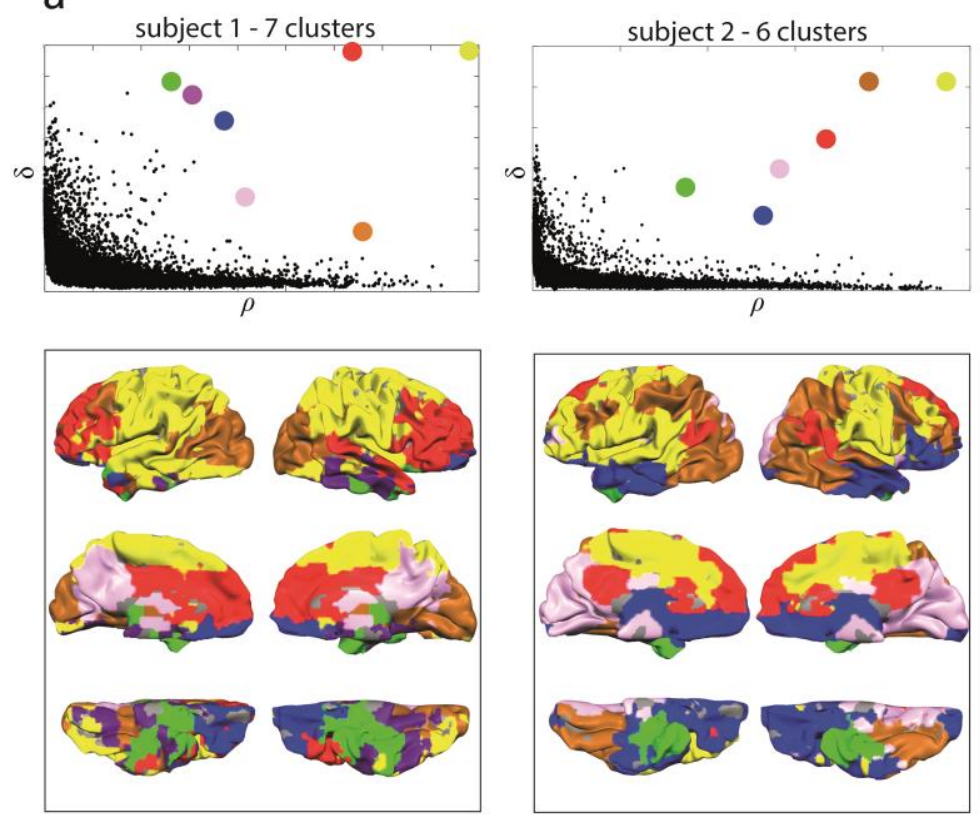

b
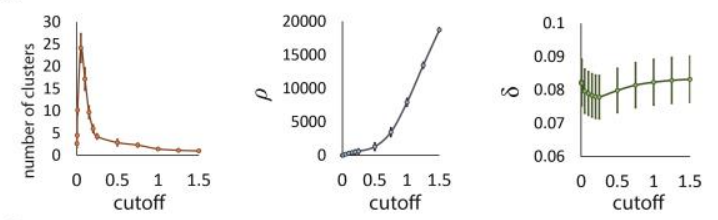

C
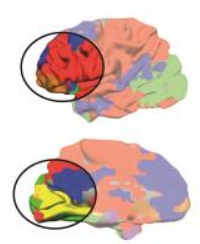

cutoff $=0.25$

Robust assignment maps cutoff $=0.25$

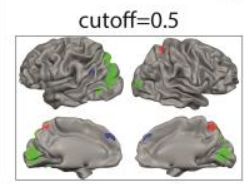

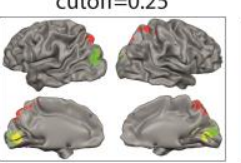

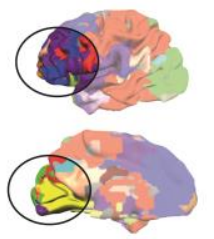

cutoff $=0.1$ cutoff $=0.1$

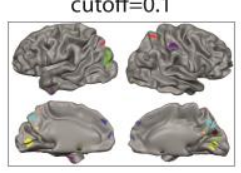

Fig. 2. (a) Clustering maps and decision graphs of two individual's resting-state data. Top: Colored points in the decision graphs denote cluster centers (outliers). Bottom: all voxels were assigned to some cluster.. (b) Features of the algorithm across a range of distance cutoffs. Cutoff values are percent from whole brain mean correlation (e.g. when cutoff $=0.5$, the radius for density calculation is half of the mean correlation across all voxel pairs in brain; See Materials and Methods for details). Error bars denote variability across subjects $(N=23)$. (c) Single subject clustering maps during resting state using different cutoffs reveals hierarchical parcellation (black circles) - big clusters were divided to smaller cluster when decreasing cutoff. (d) Robust core maps - single subject clustering maps when not all voxels are assigned to a cluster. Only voxels within correlation distance smaller than the cutoff distance are colored according to the assigned clusters (See Materials and Methods). 


\section{References}

Damoiseaux JS, Rombouts S, Barkhof F, Scheltens P, Stam CJ, Smith SM, Beckmann $\mathrm{CF}$. Consistent resting-state networks across healthy subjects. Proceedings of the national academy of sciences, 2006; 103: 13848-53.

Esposito F, Scarabino T, Hyvarinen A, Himberg J, Formisano E, Comani S, Tedeschi G, Goebel R, Seifritz E, Di Salle F. Independent component analysis of fMRI group studies by self-organizing clustering. Neuroimage, 2005; 25: 193-205.

Hagmann P, Cammoun L, Gigandet X, Meuli R, Honey CJ, Wedeen VJ, Sporns O. Mapping the structural core of human cerebral cortex. PLoS Biol, 2008; 6: e159.

Herbet G, Duffau $\mathrm{H}$. Revisiting the functional anatomy of the human brain: Toward a meta-networking theory of cerebral functions. Physiological Reviews, 2020; 100: 1181228.

Ipiña IP, Perl YS, Kringelbach ML, Kehoe PD, Deco G, Laufs H, Tagliazucchi E. Modeling the relationship between regional activation and functional connectivity during wakefulness and sleep. arXiv preprint arXiv:1907.04412, 2019.

Ji JL, Spronk M, Kulkarni K, Repovš G, Anticevic A, Cole MW. Mapping the human brain's cortical-subcortical functional network organization. Neuroimage, 2019; 185: 3557.

Power JD, Cohen AL, Nelson SM, Wig GS, Barnes KA, Church JA, Vogel AC, Laumann TO, Miezin FM, Schlaggar BL. Functional network organization of the human brain. Neuron, 2011; 72: 665-78.

Qiu X, Mao Q, Tang Y, Wang L, Chawla R, Pliner HA, Trapnell C. Reversed graph embedding resolves complex single-cell trajectories. Nature methods, 2017; 14: 979. Rodriguez A, Laio A. Clustering by fast search and find of density peaks. Science, 2014; 344: $1492-6$.

Smith SM, Fox PT, Miller KL, Glahn DC, Fox PM, Mackay CE, Filippini N, Watkins KE, Toro R, Laird AR. Correspondence of the brain's functional architecture during activation and rest. Proceedings of the national academy of sciences, 2009; 106 : 13040-5.

Sohn WS, Yoo K, Lee Y-B, Seo SW, Na DL, Jeong Y. Influence of ROI selection on resting state functional connectivity: an individualized approach for resting state fMRI analysis. Frontiers in neuroscience, 2015; 9: 280.

Van Den Heuvel MP, Pol HEH. Exploring the brain network: a review on resting-state fMRI functional connectivity. European neuropsychopharmacology, 2010; 20: 519-34. Wang S, Wang D, Li Y. Comment on "Clustering by fast search and find of density peaks". arXiv preprint arXiv:1501.04267, 2015.

Wig GS, Laumann TO, Cohen AL, Power JD, Nelson SM, Glasser MF, Miezin FM, Snyder AZ, Schlaggar BL, Petersen SE. Parcellating an individual subject's cortical and subcortical brain structures using snowball sampling of resting-state correlations. Cerebral cortex, 2014; 24: 2036-54.

Zilles K, Amunts K. Centenary of Brodmann's map-conception and fate. Nature Reviews Neuroscience, 2010; 11: 139-45. 